Case

Report

\title{
Two Cases of Endovascular Repair with the Stent Graft for Retrograde Type A Acute Aortic Dissection with Complications
}

\author{
Takahiko Masuda, MD, Masaki Hata, MD, Kazuhiro Yamaya, MD, \\ Tomoyuki Suzuki, MD, PhD, and Naoya Terao, MD
}

\begin{abstract}
Thoracic endovascular aortic repair (TEVAR) is used to treat retrograde type A acute aortic dissection (RTAAD). In case 1, a 52-year-old man, who was conservatively managed, reported worsening back pain. Emergency TEVAR was performed 7 days after onset. After deploying two GORE Conformable TAG (CTAG) in the descending aorta, his symptoms disappeared. In case 2, a 52-year-old man with progressive worsening resistant hypertension, renal dysfunction, and respiratory failure despite maximal medical therapy underwent TEVAR 8 days after onset. A CTAG was deployed from the left subclavian artery under rapid pacing, and two Zenith Dissection stents were placed, which resolved complications. In both cases, after 6 months, computed tomography (CT) scan showed complete resorption of the false lumen in the ascending aorta. TEVAR for RTAAD with complete thrombosis of false lumen in the ascending aorta can be an alternative to surgery when the primary tear is located in descending aorta.
\end{abstract}

Keywords: Type A acute aortic dissection, retrograde type A aortic dissection, thoracic endovascular aortic repair (TEVAR)

\section{Introduction}

The treatment of retrograde type A acute aortic dissection (RTAAD) with a primary intimal tear in the descending aorta remains controversial. RTAAD accounts for $7 \%-25 \%$ of all cases of acute type A aortic dissection. ${ }^{1)}$ RTAAD is associated with a high operative mortality and morbidity because it is difficult to resect a primary intimal tear in the descending aorta via median sternotomy.

Department of Cardiovascular Surgery, Sendai Kosei Hospital, Sendai, Miyagi, Japan

Received: October 27, 2017; Accepted: December 28, 2017 Corresponding author: Takahiko Masuda, MD. Department of Cardiovascular Surgery, Sendai Kosei Hospital, 4-15 Hirose-machi, Aoba-ku, Sendai, Miyagi 980-0873, Japan

Email: mmcfj813@ybb.ne.jp

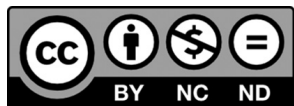

This work is licensed under a Creative Commons Attribution-NonCommercial-

NoDerivatives International License.

(C2019 The Editorial Committee of Annals of Thoracic and Cardiovascular Surgery
On the other hand, thoracic endovascular aortic repair (TEVAR) has been shown to be a safe and effective treatment for complicated type B dissection. Recently, successful results of TEVAR for RTAAD have been reported. ${ }^{2)}$ In this report, we present our experience with two cases of complicated RTAAD who were treated with TEVAR.

\section{Case Reports}

Case 1

A 52-year-old man with severe chest and back pain was referred to our hospital with a diagnosis of RTAAD based on contrast-enhanced computed tomography (CT). The CT scan showed complete thrombosis of the false lumen of the ascending aorta; the false lumen of the descending aorta was patent (Fig. 1A). He was managed conservatively because the false lumen of the ascending aorta was completely thrombosed. However, the back pain began to worsen 4 days after onset. Owing to persistence of symptoms, he was referred to our hospital and was treated 

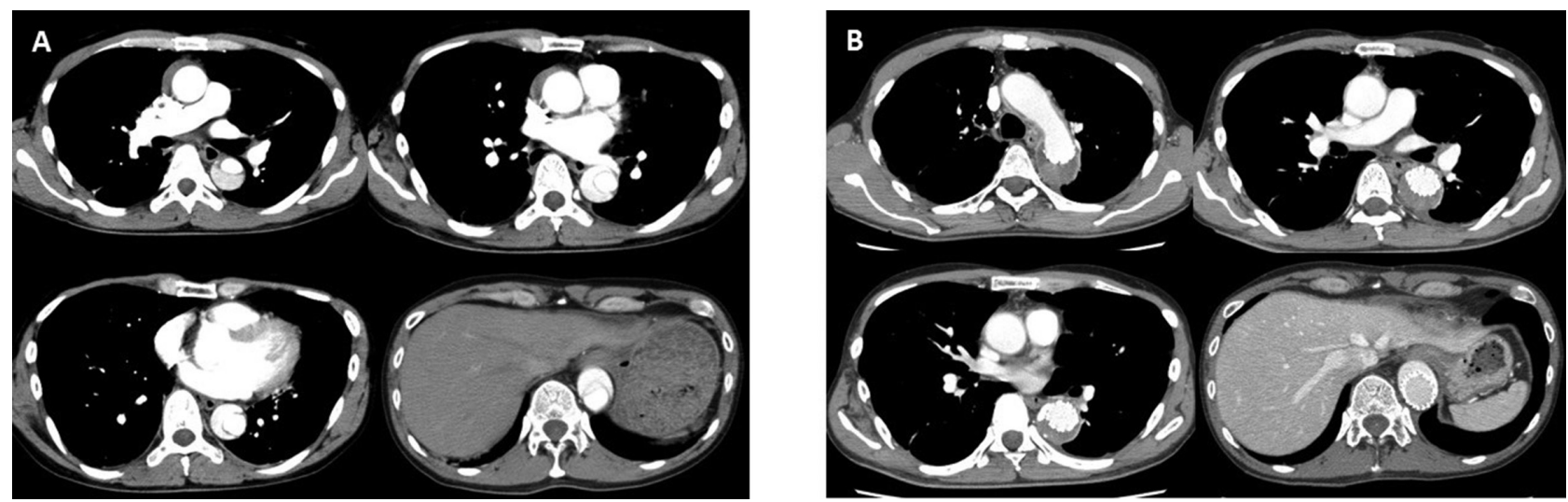

Fig. 1 Case 1: Preoperative (A) and postoperative (10 days after TEVAR) (B) CT image. (A) CT image shows complete thrombosis of the ascending aorta, the false lumen of the descending aorta with an entry tear is patent. (B) CT image shows complete resorption of the false lumen in the ascending aorta; however, residual flow into the false lumen is seen. CT: computed tomography; TEVAR: thoracic endovascular aortic repair

by emergency TEVAR 7 days after onset. The primary intimal tear seemed to be in the descending aorta $4 \mathrm{~cm}$ proximal from the origin of the celiac artery. The right femoral artery was exposed for delivery of the stent graft. To cover the entry tear, a GORE Conformable TAG (CTAG) (TGU313115J) (W. L. Gore \& Associated, Inc., Flagstaff, AZ, USA) was deployed from the celiac artery. Because of flow to false lumen from an intercostal artery, a GORE CTAG (TGU313115J) was added more proximal. Final aortography revealed no flow into the false lumen apart from the reentry flow from the iliac artery. Soon after the operation, his symptoms resolved. He was discharged on postoperative day 24 after rehabilitation. Postoperative CT scan showed regression of the false lumen in the ascending aorta (Fig. 1B). Follow-up CT scan performed 6 months after TEVAR showed complete resorption of the false lumen in the ascending aorta. However, the false lumen in the descending aorta showed incomplete thrombosis and the diameter of the distal arch was $45 \mathrm{~mm}$, which was $5 \mathrm{~mm}$ more than that at the time of TEVAR. CT scan 1 year after TEVAR showed no further expansion from that observed at 6 month after TEVAR (Fig. 2).

\section{Case 2}

A 52-year-old man presented to our hospital with chest and back pain. He was diagnosed with RTAAD based on contrast-enhanced $\mathrm{CT}$ and received conservative treatment because the false lumen of the ascending aorta was completely thrombosed and his hemodynamic status was stable. However, his condition progressively worsened following admission, he developed resistant hypertension, renal dysfunction, and respiratory failure despite maximal medical therapy. His serum creatinine level increased to
$2.15 \mathrm{mg} / \mathrm{dL}$. Respiratory failure necessitated mechanical ventilation. Since the CT scan indicated that the true lumen was severely compressed by the false lumen and the primary intimal tear was on the descending aorta (Fig. 3A), we performed TEVAR to alleviate organ malperfusion 8 days after onset. To close the entry tear, a GORE CTAG (TGU343415J) was deployed on zone 3 from the left subclavian artery under rapid pacing, and two Zenith Dissection stents (GZSD 46-164-2) (Cook Medical, Bloomington, IN, USA) were placed under the bilateral renal artery. Aortography was subsequently performed to ensure exclusion of the primary entry tear and adequate flow in the visceral and iliac arteries. After the procedure, a dramatic improvement in complications was observed. The patient was extubated on postoperative day 7 and discharged on postoperative day 24. Postoperative CT scan showed that the true lumen was expanded and the false lumen showed regression. Follow-up CT scan performed 6 months after TEVAR showed complete resorption of the false lumen of the ascending aorta. In spite of the patent false lumen in the descending aorta, the diameter of the descending aorta did not expand (Fig. 3B).

\section{Discussion}

The surgical outcomes of acute type A aortic dissection have witnessed a dramatic improvement owing to advances in surgical techniques and perioperative management. The management of RTAAD is not well established. Some studies have found favorable outcomes with conservative medical treatment in clinically stable patients in whom the false lumen of the ascending aorta is completely thrombosed. Kaji et al. ${ }^{3)}$ reported a 5-year survival rate of 

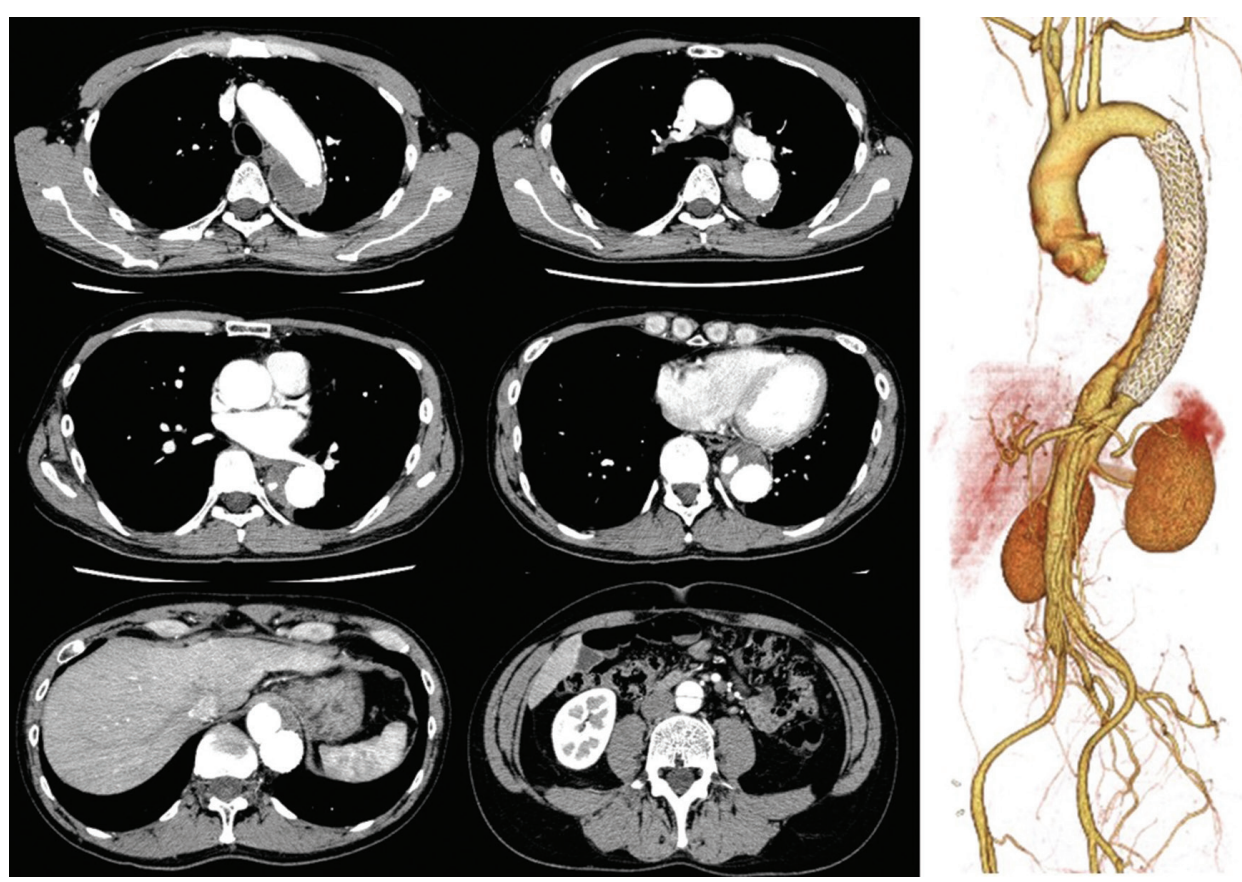

Fig. 2 Case 1: CT image 1 year after TEVAR. The false lumen in the descending aorta shows incomplete thrombosis, the diameter of the distal arch was $45 \mathrm{~mm}$, which is $5 \mathrm{~mm}$ more than that recorded immediately post-TEVAR. CT: computed tomography; TEVAR: thoracic endovascular aortic repair
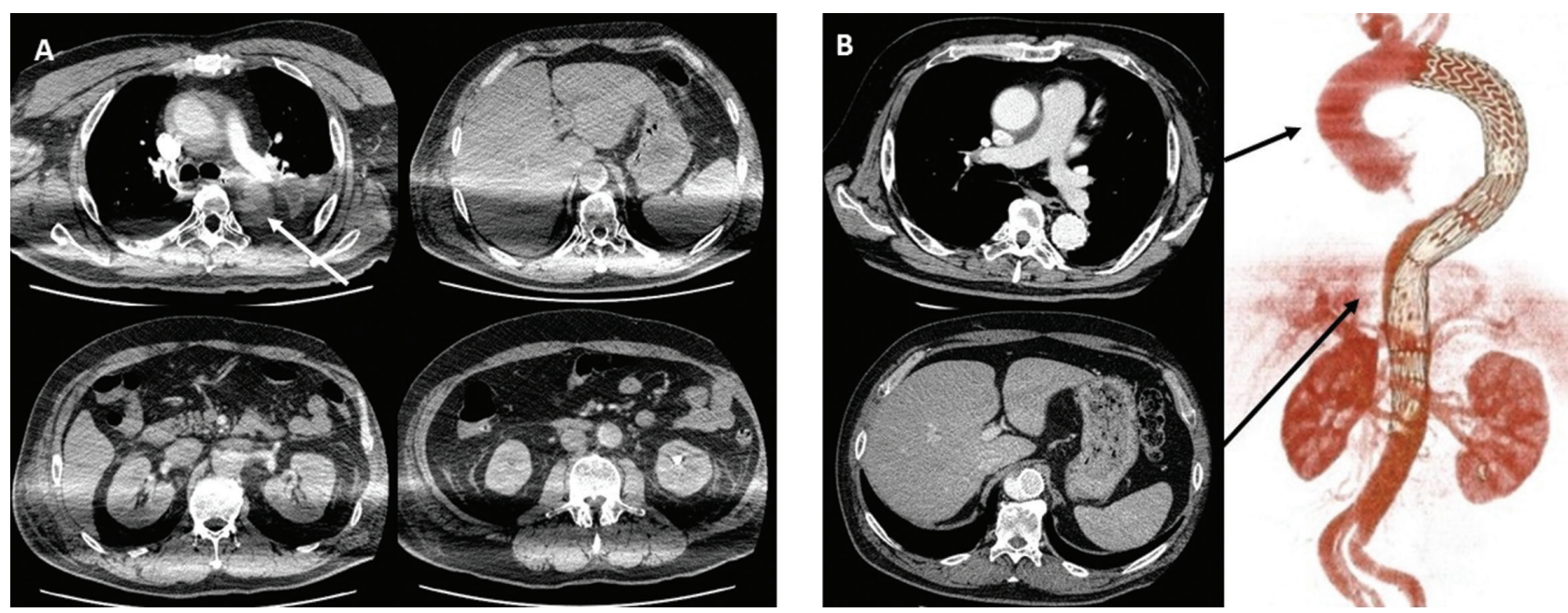

Fig. 3 Case 2: Preoperative (A) and postoperative (6 months after TEVAR) (B) CT image. (A) CT image shows significant narrowing of the true lumen. The entry tear is in the distal arch (white arrow). (B) CT image shows complete resorption of the false lumen in the ascending aorta and incomplete thrombosis of the false lumen in the descending aorta (black arrow). CT: computed tomography; TEVAR: thoracic endovascular aortic repair

$93 \%$ in a study of 14 patients with complete thrombosis of the false lumen of the ascending aorta who received optimal medical therapy. However, development of complications such as organ malperfusion, persistent pain, and rapture may necessitate surgery even in patients who show complete thrombosis of the false lumen of the ascending aorta.
RTAAD is one of the most surgically challenging type of aortic dissection. As resection of the primary tear is not easy, primary tear often remains after standard repair in many cases. Recently, the effectiveness of open stent graft (OSG) for type A aortic dissection with proximal arch replacement has been reported. Uchida ${ }^{4)}$ reported that total arch replacement with OSG improved operative 
mortality and morbidity for RTAAD. However, resection of primary intimal tear may not always be possible even with use of OSG. The patent false lumen of the descending aorta by residual entry is likely to require distal reoperation in the longer term and may cause organ malperfusion or rapture in the acute phase.

In contrast, for complicated type B acute aortic dissection, the safety and effectiveness of the entry tear closure with TEVAR is well established. ${ }^{5,6)}$ Therefore, coverage of the primary entry tear in the descending aorta with stent graft has been tried in a measured fashion in cases of RTAAD. In 1998, Shimono et al. ${ }^{7)}$ reported the first case of successful endovascular repair for RTAAD. Subsequently, there have been several reports of TEVAR for RTAAD using custom-made or commercially available stent grafts. $\left.{ }^{8}{ }^{89}\right)$ However, in these reports, most cases of TEVAR for RTAAD were performed 14 or more days after onset. Aoki et al. ${ }^{2)}$ reported the operative results of 10 consecutive cases of TEVAR for complicated RTAAD in the acute phase using TAG or CTAG. All patients in this report were complicated cases and included three patients with patent false lumen of the ascending aorta; in nine of these patients, TEVAR was performed within $24 \mathrm{~h}$ of onset. Significant remodeling of the ascending aorta was observed in all patients on follow-up CT. Over a median follow-up period of 19.5 months, no death occurred and none of the patients required reoperation. Nonetheless, there are no studies that have evaluated the mortality and morbidity associated with TEVAR for RTAAD.

In our cases, both patients received conservative treatment initially because of complete thrombosis of the false lumen of the ascending aorta and stable hemodynamics. However, occurrence of complication necessitated surgical intervention. Because the false lumen of the ascending aorta was completely thrombosed and primary intimal tear was in the descending aorta, we decided to perform TEVAR.

A key concern related to TEVAR is the risk of new tear at the proximal stent graft edge by landing on the flap and inflow to the thrombosed false lumen. Thus, we chose GORE CTAG, which have no long and strong radial force bare stents, and opted for up to $10 \%$ oversizing of stent graft based on the proximal landing zone diameter in both cases. In case 2, we deployed stent grafts under rapid pacing to decrease blood pressure during deployment. However, the effectiveness of rapid pacing to minimize the risk of new tear caused by deployment has not been demonstrated.

Soon after TEVAR, back pain disappeared in case 1, and resistant hypertension, renal dysfunction, and respiratory failure improved in case 2. Both patients were discharged after an uneventful postoperative period. TEVAR is thought to be effective for organ malperfusion and persistent pain. The most recent CT scans demonstrated disappearance of the false lumen of the ascending aorta in both cases. However, both cases showed residual flow into the false lumen from distal reentry in the descending aorta. In case 1, follow-up CT scan at 6 months after TEVAR showed that the diameter of the distal arch was $5 \mathrm{~mm}$ larger than that recorded immediately after TEVAR. Close follow-up is needed.

\section{Conclusion}

TEVAR for RTAAD with completely thrombosed false lumen in the ascending aorta is less invasive and can be an alternative to surgical treatment when the primary tear is located distal to the subclavian artery. Primary results of this strategy seem to be promising. Long-term follow-up and more reports are needed to examine the effectiveness of this strategy and to apply this approach more widely.

\section{Acknowledgment}

The authors would like to thank Enago (www.enago.jp) for the English language review.

\section{Disclosure Statement}

None of the authors have any conflicts of interest to declare.

\section{References}

1) Kim JB, Choo SJ, Kim WK, et al. Outcomes of acute retrograde type A aortic dissection with an entry tear in descending aorta. Circulation 2014; 130: S39-44.

2) Aoki K, Okamoto T, Sato H, et al. [Endovascular repair for acute phase of retrograde type A aortic dissection with an entry in the descending aorta]. Kyobu Geka 2016; 69: 276-81. (in Japanese)

3) Kaji S, Akasaka T, Katayama M, et al. Prognosis of retrograde dissection from the descending to the ascending aorta. Circulation 2003; 108: II300-6.

4) Uchida N. Open stent grafting for complex diseases of the thoracic aorta: clinical utility. Gen Thorac Cardiovasc Surg 2013; 61: 118-26.

5) Lombardi JV, Cambria RP, Nienaber CA, et al. Prospective multicenter clinical trial (STABLE) on the endovascular treatment of complicated type B aortic dissection using a composite device design. J Vasc Surg 2012; 55: 629-40. 
6) Alsac JM, Girault A, El Batti S, et al. Experience of the Zenith dissection endovascular system in the emergency setting of malperfusion in acute type B dissections. J Vasc Surg 2014; 59: 645-50.

7) Shimono T, Kato N, Tokui T, et al. Endovascular stentgraft repair for acute type A aortic dissection with an intimal tear in the descending aorta. Thorac Cardiovasc Surg 1998; 116: 171-173.
8) Dake MD, Kato N, Mitchell RS, et al. Endovascular stent-graft placement for the treatment of acute aortic dissection. N Engl J Med 1999; 340: 1546-52.

9) Kato N, Shimono T, Hirano T, et al. Transluminal placement of endovascular stent-grafts for the treatment of type A aortic dissection with an entry tear in the descending thoracic aorta. J Vasc Surg 2001; 34: 1023-8. 